\title{
Investigation of Thermal and Viscoelastic Properties of Polymers Relevant to Hot Melt Extrusion, IV: Affinisol ${ }^{\mathrm{TM}}$ HPMC HME Polymers
}

\author{
Simerdeep Singh Gupta, ${ }^{1,2}$ Nayan Solanki, ${ }^{1}$ and Abu T. M. Serajuddin ${ }^{1,3}$
}

Received 28 July 2015; accepted 28 September 2015; published online 28 October 2015

\begin{abstract}
Most cellulosic polymers cannot be used as carriers for preparing solid dispersion of drugs by hot melt extrusion (HME) due to their high melt viscosity and thermal degradation at high processing temperatures. Three HME-grade hydroxypropyl methylcelluloses, namely Affinisol ${ }^{\mathrm{TM}}$ HPMC HME $15 \mathrm{cP}$, Affinisol ${ }^{\mathrm{TM}}$ HPMC HME $100 \mathrm{cP}$, and Affinisol ${ }^{\mathrm{TM}}$ HPMC HME $4 \mathrm{M}$, have recently been introduced by The Dow Chemical Co. to enable the preparation of solid dispersion at lower and more acceptable processing temperatures. In the present investigation, physicochemical properties of the new polymers relevant to HME were determined and compared with that of Kollidon ${ }^{\circledR}$ VA 64. Powder X-ray diffraction (PXRD), modulated differential scanning calorimetry (mDSC), thermogravimetric analysis (TGA), moisture sorption, rheology, and torque analysis by melt extrusion were applied. PXRD and mDSC showed that the Affinisol ${ }^{\mathrm{TM}}$ polymers were amorphous in nature. According to TGA, the onset of degradation for all polymers was $>220^{\circ} \mathrm{C}$. The Affinisol ${ }^{\mathrm{TM}}$ polymers exhibited less hygroscopicity than Kollidon ${ }^{\circledR}$ VA 64 and another HPMC polymer, Methocel ${ }^{\mathrm{TM}} \mathrm{K} 100 \mathrm{LV}$. The complex viscosity profiles of the Affinisol ${ }^{\mathrm{TM}}$ polymers as a function of temperature were similar. The viscosity of the Affinisol ${ }^{\mathrm{TM}}$ polymers was highly sensitive to the shear rate applied, and unlike Kollidon ${ }^{\circledR}$ VA 64, the viscosity decreased drastically when the angular frequency was increased. Because of the very high shear rate encountered during melt extrusion, Affinisol ${ }^{\mathrm{TM}}$ polymers showed capability of being extruded at larger windows of processing temperatures as compared to that of Kollidon ${ }^{\circledR}$ VA 64.
\end{abstract}

KEY WORDS: Affinisol ${ }^{\mathrm{TM}}$ HPMC HME; hot melt extrusion; hydroxypropyl methylcellulose; solid dispersion; thermal analysis; viscosity.

\section{INTRODUCTION}

Poor aqueous solubility of drugs has emerged as a major challenge for formulation scientists for the development of oral drug products (1). It has been estimated that two out of three new chemical entities (NCE) synthesized in the pharmaceutical industry have an aqueous solubility of $<0.1 \mathrm{mg} / \mathrm{mL}$ and, therefore, may be classified, according to the definition of the United States Pharmacopeia, as insoluble or practically insoluble in aqueous media (2). Even a large fraction of the remaining one-third of the compounds with the solubility of $>0.1 \mathrm{mg} / \mathrm{mL}$ may be considered to have low solubility according to the Biopharmaceutical Classification System (BCS), because the required dose may not dissolve in $250 \mathrm{~mL}$ of a dissolution

\footnotetext{
${ }^{1}$ Department of Pharmaceutical Sciences, College of Pharmacy and Health Sciences, St. John's University, 8000 Utopia Parkway, Queens, New York 11439, USA.

${ }^{2}$ Present Address Teva Pharmaceuticals Inc., 223 Quaker Rd, Pomona, New York 10970, USA.

${ }^{3}$ To whom correspondence should be addressed. (e-mail: serajuda@stjohns.edu)
}

medium in the gastrointestinal $\mathrm{pH}$ range (3). After oral administration, the low aqueous solubility often leads to slow dissolution rate, incomplete absorption, and low bioavailability of drugs (4).

Among various formulation strategies applied to overcome development issues with poorly water-soluble drugs, the amorphous solid dispersion, where the drug is dispersed in a polymeric matrix either molecularly or in the amorphous state, has emerged as one of the most promising options $(5,6)$. However, various issues related to formulation, manufacturing, and physical stability of solid dispersions have for a long time hindered its widespread application in drug product development (7). The situation has changed greatly in the past decade because of the introduction of hot melt extrusion (HME) for the preparation of solid dispersion (8-11). In HME, the drug is solubilized in a polymeric matrix by processing blends of drug, polymer, and any other excipients at high temperatures in a hot melt extruder (9). It is a solvent-free, continuous, and less time-consuming process that may be easily scaled up. However, being a relatively new technology in the pharmaceutical field, there is only limited knowledge on formulation and processing parameters of solid dispersion by 
HME available in the literature. In particular, the criteria for the selection of appropriate polymers for HME based on their glass transition temperature, complex viscosity, extrudability through a melt extruder, drug-polymer miscibility, and so forth are not fully understood.

We previously studied physicochemical properties of several polymers having PVP (12), cellulose (13), and methacrylate (14) backbones to investigate their suitability for melt extrusion. Although the cellulosic polymers were extensively used in the development of solid dispersion systems by using spray drying and other solvent evaporation methods because of their advantages in physically stabilizing amorphous drugs in solid dispersions, improving drug release due to better wetting and swelling, inhibiting nucleation into crystalline form and maintaining supersaturation after dissolution in aqueous media, and improving bioavailability, it was observed that most cellulosic polymers were not suitable for melt extrusion (13). This is due to high melt viscosity of cellulosic polymers, which generates high torque within the extruder during processing. Although the melt viscosity and the torque decrease with the increase in temperature, cellulosic polymers and drugs used may degrade at the relatively high temperature required to enable processing (13). To ameliorate the situation, The Dow Chemical Co. (MI, USA), which manufactures many of the cellulosic polymers, has recently made available to pharmaceutical researchers several new grades of hydroxypropyl methylcellulose (HPMC), collectively known as AffinisolTM HPMC HME, that may be extruded through hot melt extruders at lower processing temperatures as they have been tailored to have relatively lower glass transition temperatures $\left(\mathrm{T}_{\mathrm{g}}\right)$ and improved 'melt flow' (15). In addition to improving processing by HME, the new Affinisol ${ }^{\text {TM }}$ HPMC HME polymers have been claimed to maintain advantages as the precipitation inhibitor after dissolution $(16,17)$.

Despite the promising prospects of Affinisol ${ }^{\mathrm{TM}}$ HPMC HME polymers, limited information on their physicochemical properties relevant to melt extrusion has been reported in the literature. The objective of the present study was to evaluate the suitability of Affinisol ${ }^{\text {TM }}$ HPMC HME polymers for melt extrusion by investigating their thermal and viscoelastic properties. The investigation specifically included (i) determination of thermal properties, such as glass transition temperature $\left(\mathrm{T}_{\mathrm{g}}\right)$ and degradation temperature $\left(\mathrm{T}_{\mathrm{d}}\right)$, by using differential scanning calorimetry (DSC) and thermogravimetric analysis (TGA), (ii) determination of moisture sorption as a function of relative humidity, (iii) identification of physical form by powder X-ray diffraction (PXRD), (iv) evaluation of viscoelastic properties in terms of complex viscosity as a function of temperature and angular frequency, and (v) torque analysis by using a hot melt extruder. Another polymer, Kollidon ${ }^{\circledR}$ VA 64, which is a PVP-based copolymer that is commonly used in melt extrusion (18) and has similar $\mathrm{T}_{\mathrm{g}}$ value (12) as those of Affinisol ${ }^{\mathrm{TM}}$ HPMC HME polymers, was also studied to establish a benchmark for comparing physicochemical properties of the new polymers. Additionally, the moisture sorption of the AffinisolTM HPMC HME polymers was compared with that of a marketed HPMC polymer (HPMC $100 \mathrm{cP}$; Methocel ${ }^{\mathrm{TM}}$ K100LV) available from The Dow Chemical Co., whose physicochemical properties relevant to melt extrusion, except for the moisture sorption, were reported earlier (13).

\section{MATERIALS AND METHODS}

\section{Materials}

Three Affinisol ${ }^{\mathrm{TM}}$ HPMC HME polymers, namely Affinisol $^{\mathrm{TM}} 15 \mathrm{cP}$, Affinisol ${ }^{\mathrm{TM}} 100 \mathrm{cP}$, and Affinisol ${ }^{\mathrm{TM}} 4 \mathrm{M}$ (4000 cP), were donated by The Dow Chemical Co., MI, USA. They were received in February 2015, and according to the manufacturer, they were from batches prepared for distribution to researchers as experimental samples. Methocel $^{\mathrm{TM}}$ K100LV was also received from The Dow Chemical Co. According to the personal communication from the manufacturer, Affinisol ${ }^{\mathrm{TM}}$ HPMC HME polymers are pure hydroxypropyl methylcellulose (HPMC) without any additives present, and they differ among themselves and from other HPMC polymers, such as Methocel ${ }^{\mathrm{TM}} \mathrm{K} 100 \mathrm{LV}$, in the level of chemical substitution. The polyvinylpyrrolidone-vinyl acetate copolymer, Kollidon ${ }^{\circledR}$ VA 64 , was donated by BASF, Tarrytown, NY, USA. All materials were used as received.

\section{Methods}

\section{Powder X-ray Diffraction}

PXRD patterns of polymers were obtained using a Shimadzu XRD-6000 diffractometer (Shimadzu, Kyoto, Japan), equipped with $\mathrm{Ni}$ filtered $\mathrm{Cu}-\mathrm{K} \alpha$ as the $\mathrm{x}$-ray source. The diffractometer was operated with a copper anode tube at generator voltage and current of $40 \mathrm{kV}$ and $30 \mathrm{~mA}$, respectively. The 2-theta scanning range was from $10^{\circ}$ to $60^{\circ}$ at a rate of $2^{\circ}$ per minute. The polymers were gently grinded using agate mortar and pestle before loading onto the glass sample holder and leveling with a glass plate.

\section{Modulated Differential Scanning Calorimetry (mDSC)}

The modulated DSC (mDSC) scans were recorded using a Q200 modulated DSC analyzer (TA instruments, DE, USA). Sample was weighed $(\sim 5 \mathrm{mg}$ each) and sealed in Tzero ${ }^{\circledR}$ aluminum pan. The pan was then equilibrate at $25^{\circ} \mathrm{C}$ for $3 \mathrm{~min}$, which was followed by heating to $200^{\circ} \mathrm{C}$ at the ramp rate of $3^{\circ} \mathrm{C} / \mathrm{min}$ and modulation of $1^{\circ} \mathrm{C} / \mathrm{min}$. The $\mathrm{T}_{\mathrm{g}}$ of a polymer was obtained by deconvolution of total heat flow into nonreversible and reversible phenomena using Universal Analysis software (TA instruments, DE, USA).

\section{Thermogravimetric Analysis}

TGA of polymers was performed to determine the degradation temperature $\left(\mathrm{T}_{\mathrm{d}}\right)$ using a thermogravimetric analyzer, TGA Q50 (TA instruments, DE, USA). For analysis, $5 \mathrm{mg}$ of sample was weighed into a tared crucible and equilibrated for $30 \mathrm{~min}$ at room temperature under a constant nitrogen purge, which was followed by heating up to $300^{\circ} \mathrm{C}$ at a rate of $5^{\circ} \mathrm{C} / \mathrm{min}$.

\section{Moisture Sorption}

An automated moisture sorption analyzer VTI-SA ${ }^{+}$(TA instruments, DE, USA), equipped with a specially designed climatic chamber and an analytical balance with a resolution 
of $0.01 \mu \mathrm{g}$, was used. The moisture sorption (or desorption) at $25.0 \pm 0.1^{\circ} \mathrm{C}$ was determined in the relative humidity range of 10 to $90 \%$ RH. An accurately weighed sample of each polymer used ( 20 mg) was uniformly spread in a platinum pan and placed in the moisture sorption analyzer. The equilibrium condition was set to $<0.01 \%$ change in the weight for $5 \mathrm{~min}$ within a period of $120 \mathrm{~min}$ at each humidity condition. The sample was first equilibrated at $10 \% \mathrm{RH}$, and the relative humidity was automatically increased by intervals of $10 \%$ $\mathrm{RH}$ and up to $90 \% \mathrm{RH}$, when the equilibrium condition at each $\mathrm{RH}$ was reached. The equipment recorded the weight change of a sample every $\geq 0.001 \%$ or at every minute when there was no weight change.

\section{Rheology}

Rheological analysis of polymers was performed to evaluate their viscoelastic properties at various temperatures and angular frequencies. The study was carried out by a Discovery hybrid rheometer-2 (DHR-2) (TA instruments, DE, USA) with an oven heating assembly using the $25-\mathrm{mm}$ parallel plate geometry. A 500-mg slug of each polymer was prepared, according to the method described earlier (12), using a Carver press at $5000 \mathrm{lb}$ of compression pressure and the dwell time of $5 \mathrm{~s}$. The rheometer was calibrated for zero gap before analyzing a sample. Viscoelastic analyses of polymers were performed in two different ways: (i) oscillation temperature sweep, where the change in complex viscosity as a function of temperature was measured, and (ii) oscillation frequency sweep, where the effect of shear rate on the complex viscosity of a polymer at different temperatures was determined. The temperature sweep was performed from 180 to $90^{\circ} \mathrm{C}$ at the rate of $5^{\circ} \mathrm{C} / \mathrm{min}$, applied strain of $0.5 \%$ and angular frequency at $0.1^{\circ} \mathrm{radian} / \mathrm{s}$. The oscillation frequency sweep was performed at intervals of $10^{\circ} \mathrm{C}$ in the range of 100 to $180^{\circ} \mathrm{C}$ by increasing the angular frequency from 0.1 to $100^{\circ} \mathrm{radian} / \mathrm{s}$ at each temperature and using the applied strain of $0.5 \%$.

\section{Torque Analysis by Hot Melt Extruder}

The torque analyses at three different screw designs were conducted using Process 11 twin screw melt extruder (Thermo Scientific, Bridgewater, NJ, USA) at constant temperatures. The highest temperature used for torque analysis was $180^{\circ} \mathrm{C}$, and other experiments were conducted at lower temperatures at intervals of $10^{\circ} \mathrm{C}$. The lowest temperature that could be used for torque analysis depended on the extrudability of the polymer. The extruder had a maximum torque limit of about $12 \mathrm{Nm}$, which is considered to be $\sim 100 \%$ of its capacity, above which the equipment stopped. Therefore, for the proper functioning of the equipment, the lowest temperature for torque analysis was selected such that the torque remained below $90 \%$.

Three different types of screw configurations, namely low shear stress (L), medium shear stress (M), and high shear stress $(\mathrm{H})$, were selected to assess the effect of shear stress exerted by screw configuration on the extrudability of polymers. All three screw designs contained three series of conveying elements and two kneading zones. The two kneading zones consisted of 13 kneading elements each in the forward direction having different configurations in their angles: The low shear stress screw design consisted of 13 kneading elements with the successive angle of $30^{\circ}$; the medium shear stress screw design consists of six kneading elements of the successive angle of $60^{\circ}$, followed by seven kneading elements of the successive angle of $30^{\circ}$; and the high shear screw design consists of six kneading elements of the successive angle of $90^{\circ}$, followed by seven kneading elements of the successive angle of $30^{\circ}$. Throughout the experiments, the feeding rate, the screw speed, and the die diameter were kept constant, which were, respectively, $2 \mathrm{~g} / \mathrm{min}, 200 \mathrm{RPM}$, and $2 \mathrm{~mm}$.

\section{RESULTS AND DISCUSSION}

\section{PXRD}

Powder X-ray diffraction patterns of the Affinisol ${ }^{\mathrm{TM}}$ HPMC HME polymers and Kollidon ${ }^{\circledR}$ VA 64 are shown in Fig. 1. A single halo at approximately $202 \theta$ was observed for the three Affinisol ${ }^{\mathrm{TM}}$ HPMC polymers, while Kollidon ${ }^{\circledR}$ VA 64 exhibited two amorphous halos around 12 and 22 20. There were no characteristic major peaks indicating crystallinity of any material, and therefore, it was concluded based on PXRD that all polymers were amorphous.

It may be noticed in Fig. 1 that there was a minor peak at about $322 \theta$ in the PXRD pattern of each Affinisol ${ }^{\mathrm{TM}}$ HPMC HME polymer. In case of Affinisol ${ }^{\mathrm{TM}} 15 \mathrm{cP}$, a few other minor peaks at higher $2 \theta$ values were also observed. Previously, small peaks at about $322 \theta$ for HPMC 100 cps (Methocel $^{\mathrm{TM}}$ K100LV) and HPMC 6 cps were reported (13). Although the substituted polymers like HPMC are mostly amorphous, a small degree of crystallinity may still exist in their structures due to intra- and inter-molecular hydrogen bonding between polymer chains $(13,19)$. It is possible that the minor peaks observed in Fig. 1 are due to the presence of such microcrystalline domain in the structures of Affinisol ${ }^{\mathrm{TM}}$ HPMC HME polymers.

\section{mDSC}

Modulated differential scanning calorimetric scans of the polymers used are shown in Fig. 2. They exhibited glass transition temperatures within a relatively narrow range of 98 to $108^{\circ} \mathrm{C}$, which were $98,103,104$, and $108^{\circ} \mathrm{C}$ for Affinisol ${ }^{\mathrm{TM}}$ $15 \mathrm{cP}$, Affinisol ${ }^{\mathrm{TM}} 100 \mathrm{cP}$, Affinisol ${ }^{\mathrm{TM}} 4 \mathrm{M}$, and Kollidon ${ }^{\circledR} \mathrm{VA}$ 64 , respectively. The absence of any other thermal events confirmed that the materials were amorphous.

\section{TGA}

The results of the thermogravimetric analysis of the Affinisol ${ }^{\mathrm{TM}}$ HPMC HME polymers and Kollidon ${ }^{\circledR}$ VA 64 are shown on Fig. 3. In addition to these polymers, the TGA scan of a marketed polymer, HPMC $100 \mathrm{cP}$ (Methocel ${ }^{\mathrm{TM}} \mathrm{K} 100 \mathrm{LV}$ ), is also included in the figure from a previous study (13) for comparison with the new HPMC HME (Affinisol ${ }^{\mathrm{TM}}$ ) polymers. All five polymers exhibited rapid weight loss above $240^{\circ} \mathrm{C}$, indicating chemical degradation that produced volatiles. However, there appeared to be a gradual weight loss of Affinisol ${ }^{\mathrm{TM}} 15 \mathrm{cP}$ starting from about $100^{\circ} \mathrm{C}$ and there also appeared to be some stepwise weight loss of Affinisol ${ }^{\mathrm{TM}}$ $100 \mathrm{cP}$ and Affinisol ${ }^{\mathrm{TM}} 4 \mathrm{M}$ starting at about $190^{\circ} \mathrm{C}$. For this 


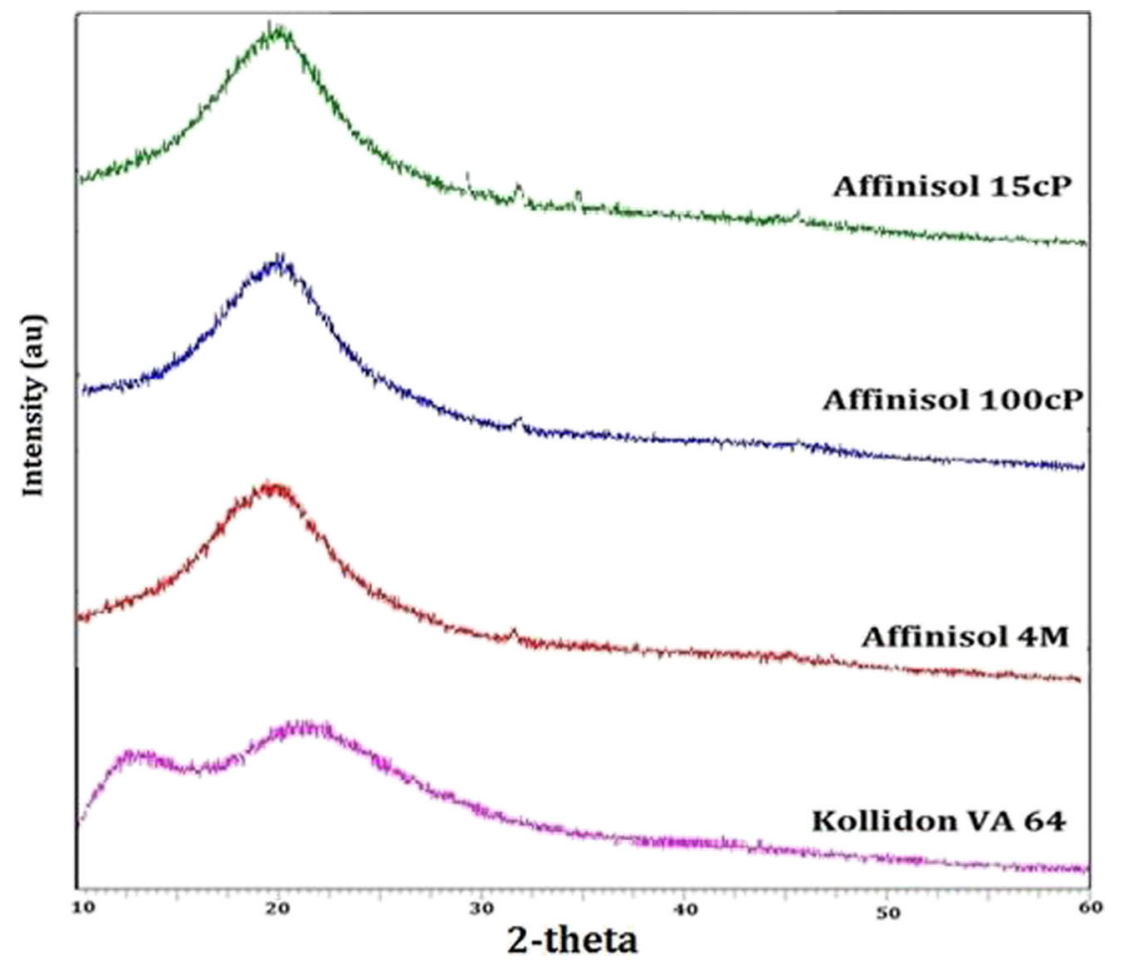

Fig. 1. Powder XRD patterns of Affinisol ${ }^{\mathrm{TM}}$ polymers and Kollidon ${ }^{\circledR}$ VA 64

reason, weight losses by the polymers at different temperature ranges are tabulated in Table $\mathrm{I}$. The initial weight loss at $<60^{\circ} \mathrm{C}$ in Fig. 3 by Kollidon ${ }^{\circledR}$ VA 64 and Methocel ${ }^{\mathrm{TM}}$ $\mathrm{K} 100 \mathrm{LV}$ was attributed to the evaporation of adsorbed moisture and after that there were minimal weight loss until possible degradation at $>220^{\circ} \mathrm{C}$. For example, between 100 and $190^{\circ} \mathrm{C}$, the weight loss by Kollidon ${ }^{\circledR}$ VA 64 and Methocel $^{\mathrm{TM}}$ K100LV was, respectively, 0.25 and $0.20 \%$. On the other hand, the three Affinisol ${ }^{\mathrm{TM}}$ polymers showed weight loss of 0.51 to $0.65 \%$ within this temperature range. Additionally, Affinisol $^{\mathrm{TM}} 100 \mathrm{cP}$ and Affinisol ${ }^{\mathrm{TM}} 4 \mathrm{M}$ showed weight loss of 0.59 and $0.50 \%$ between 190 and $220^{\circ} \mathrm{C}$, as compared to the weight loss of 0.21 and $0.25 \%$ within the same range of temperature by Kollidon ${ }^{\circledR}$ VA 64 and Methocel $^{\text {TM }}$ K100LV, respectively. Overall, the three AffinisolTM HPMC HME polymers exhibited higher weight loss than those of Kollidon ${ }^{\circledR}$

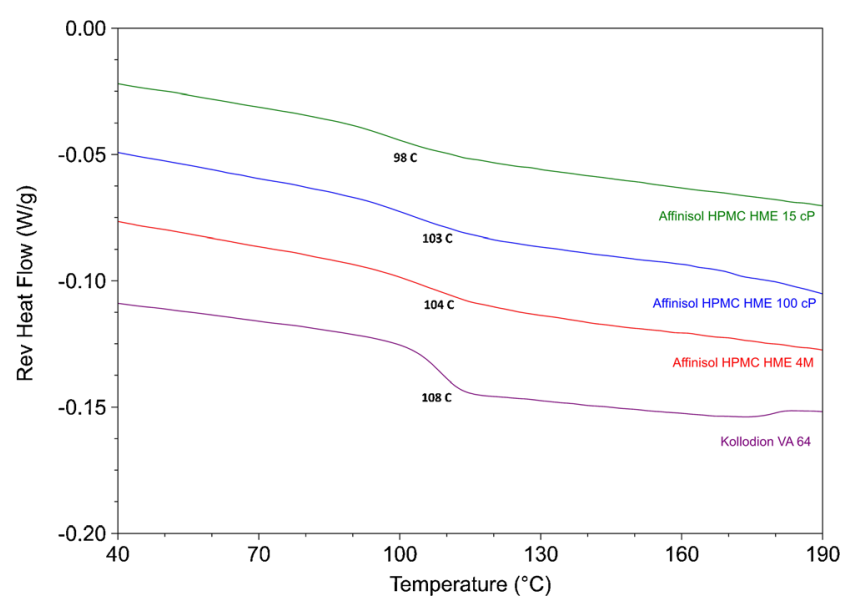

Fig. 2. DSC scans of Affinisol ${ }^{\mathrm{TM}}$ polymers and Kollidon ${ }^{\circledR}$ VA 64 showed their glass transition temperatures $\left(\mathrm{T}_{\mathrm{g}}\right)$
VA 64 and Methocel ${ }^{\mathrm{TM}} \mathrm{K} 100 \mathrm{LV}$ in the temperature range of 100 to $220^{\circ} \mathrm{C}$. Above $220^{\circ} \mathrm{C}$, all polymers exhibited comparable weight loss, possibly due to the onset of thermal degradation for all of them.

The reason for higher weight loss, although relatively small, from the Affinisol ${ }^{\mathrm{TM}}$ polymers as a function of temperature within the possible melt extrusion range of 100 to $200^{\circ} \mathrm{C}$ has not been investigated in the present study. Additional information about chemical compositions of the Affinisol ${ }^{\mathrm{TM}}$ polymers will be necessary to determine what types of volatile

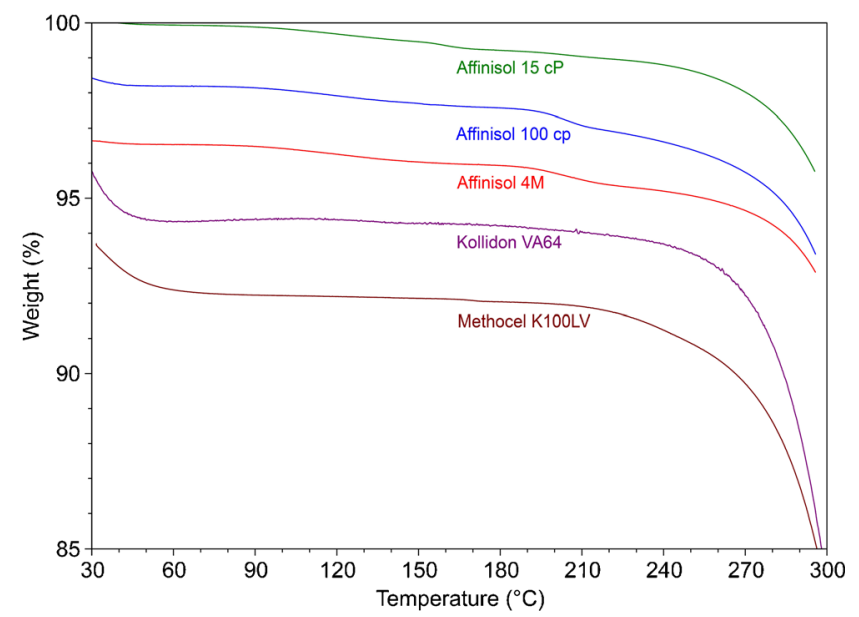

Fig. 3. TGA scans of Affinisol ${ }^{\mathrm{TM}}$ polymers, Kollidon ${ }^{\circledR}$ VA 64, and Methocel $^{\mathrm{TM}}$ K100LV. The initial weight of Affinisol ${ }^{\mathrm{TM}}$ HPMC HME $15 \mathrm{cP}$ shows $100 \%$. Although the initial weights of other polymers in the figure were also set at $100 \%$, the initial numbers in the $y$-axis do not reflect $100 \%$ as all scans are shown separately in one figure for better clarity. The weight losses in all figures are, however, proportional 
Table I. Percent Weight Loss by Different Polymers at Specified Temperature Ranges

\begin{tabular}{lccccc}
\hline Temperature range $\left({ }^{\circ} \mathrm{C}\right)$ & \multicolumn{2}{l}{ Weight loss $(\%)$} & & \\
\cline { 2 - 6 } & Affinisol $15 \mathrm{cP}$ & Affinisol $100 \mathrm{cP}$ & Affinisol 4 M & Kollidon VA 64 & Methocel K100LV \\
\hline 30 to 100 & 0.17 & 0.43 & 0.19 & 0.94 & 0.25 \\
100 to 190 & 0.65 & 0.57 & 0.51 & 0.21 & 0.20 \\
190 to 220 & 0.20 & 0.59 & 0.50 & 0.94 \\
220 to 250 & 1.01 & 1.40 & 1.14 & 1.37 \\
\hline
\end{tabular}

materials may be present. The chemical analyses of the volatiles may also be required.

\section{Moisture Sorption}

Although the primary focus of the present investigation was to study thermal and viscoelastic properties of polymers, it was of interest to compare hygroscopicity of different polymers as it may play an important role in the selection of a polymer for the preparation of solid dispersion by melt extrusion. It is well-known that the hygroscopicity of polymeric materials and other excipients used plays critical roles on physical stability of amorphous drugs present in solid dispersions as the materials may convert to crystalline forms more easily at higher moisture contents (20). Figure 4 shows moisture sorption profiles of Affinisol ${ }^{\mathrm{TM}}$ polymers, Kollidon ${ }^{\circledR}$ VA 64, and Methocel ${ }^{\mathrm{TM}} \mathrm{K} 100 \mathrm{LV}$ as a function of relative humidity at $25^{\circ} \mathrm{C}$. In this study, the samples were initially equilibrated at $10 \% \mathrm{RH}$, where there was some weight loss due to desorption of any moisture initially present with the polymers. As the humidity was increased from $10 \% \mathrm{RH}$, weight gain due to moisture sorption was observed. The extents of weight gain at 60,70 , and $80 \% \mathrm{RH}$ by different polymers relative to that at $10 \% \mathrm{RH}$ are given in Table II. Both Fig. 4 and Table II show that all Affinisol ${ }^{\mathrm{TM}}$ HPMC HME polymers were much less hygroscopic than Kollidon ${ }^{\circledR}$ VA 64 and Methocel ${ }^{\mathrm{TM}}$ K100LV.

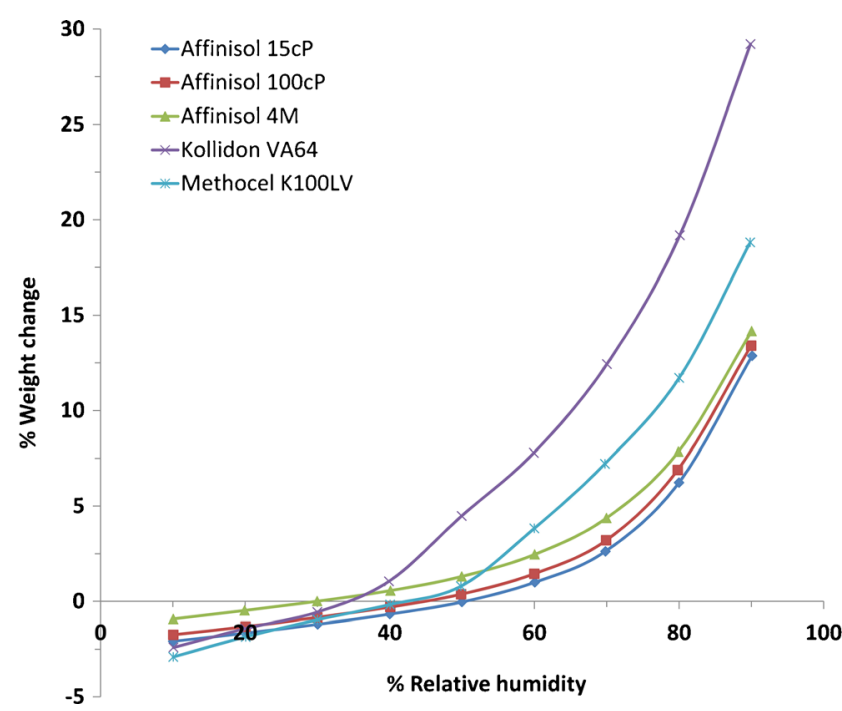

Fig. 4. Moisture sorption analysis of Affinisol ${ }^{\mathrm{TM}}$ polymers, Kollidon ${ }^{\circledR}$ VA 64, and Methocel ${ }^{\mathrm{TM}} \mathrm{K} 100 \mathrm{LV}$ where the percent weight loss or weight gain is plotted as a function of percent relative humidity at $25^{\circ} \mathrm{C}$

\section{Viscoelastic Properties}

In general, polymers are viscoelastic materials, which behave both solid-like and liquid-like and thereby exhibit deformation and flow depending on temperature and shear rate conditions. The viscoelastic properties are characteristics of a given polymer that depend on its molecular weight, molecular weight distribution, and branching. It is, therefore, important to investigate such properties of polymers by rheology prior to their use in hot melt extrusion $(12,21)$. Since the hot melt extrusion technique imparts temperature, shear rate, and stress onto materials, the rheological data of polymers helps formulation scientists to determine optimal processing conditions in the development of solid dispersions.

Among various viscoelastic properties, the complex viscosity is most relevant to melt extrusion of polymers $(12,22)$. It is obtained when the complex modulus of a polymer, which is a measure of the overall resistance of a material to deformation regardless of whether the deformation is recoverable (elastic) or nonreversible (viscous), is divided by angular frequency applied to the material in the oscillation rheology technique. The complex viscosity of polymers drops with an increase in temperature. For materials that exhibit Newtonian behavior, the viscosity as a function of shear rate at a particular temperature remains constant (constant viscosity fluids). However, polymers usually exhibit non-Newtonian shear-thinning viscoelastic properties and, as a consequence, the complex viscosity of their melts decreases with an increase in shear rate. During the measurement of viscosity by the oscillation rheology technique, an increase in angular frequency is analogous to an increase in shear rate, and therefore, the complex viscosity may also be considered to be the angular frequencydependent viscosity. High temperature is used during melt extrusion, and at the same time, the materials are subjected to high shear rate due to rotation of screws at high speed and

Table II. Percent Weight Gains at $25^{\circ} \mathrm{C}$ by Affinisol ${ }^{\mathrm{TM}}$ Polymers, Kollidon ${ }^{\circledR}$ VA 64 , and Methocel ${ }^{\mathrm{TM}} \mathrm{K} 100 \mathrm{LV}$ at 60,70 , and $80 \%$ RH Relative to the Equilibrium Weights at $10 \% \mathrm{RH}$

\begin{tabular}{lccc}
\hline Polymer & \multicolumn{3}{c}{$\%$ Weight gain at $25^{\circ} \mathrm{C}$} \\
\cline { 2 - 4 } & $60 \% \mathrm{RH}$ & $70 \% \mathrm{RH}$ & $80 \% \mathrm{RH}$ \\
\hline Affinisol 15 cP & 3.1 & 4.7 & 8.3 \\
Affinisol 100 cP & 3.2 & 4.9 & 8.6 \\
Affinisol 4 M & 3.4 & 5.3 & 8.8 \\
Kollidon VA 64 & 10.2 & 14.9 & 21.6 \\
Methocel K100LV & 6.7 & 10.1 & 14.6 \\
\hline
\end{tabular}


friction with the barrel wall. Therefore, it is essential that the effects of both temperature and angular frequency (i.e., shear rate) on complex viscosity of polymers are measured.

\section{Effect of Temperature on Complex Viscosity}

Figure 5 shows the effect of temperature on the complex viscosity of the Affinisol ${ }^{\mathrm{TM}}$ HPMC HME polymers and Kollidon ${ }^{\circledR}$ VA 64. All Affinisol ${ }^{\mathrm{TM}}$ polymers showed almost identical plots as a function of temperature at an angular frequency of $0.1 \mathrm{rad} / \mathrm{s}$. The viscosity near the $\mathrm{T}_{\mathrm{g}}$ values $\left(\sim 100^{\circ} \mathrm{C}\right)$ of the polymers was above $1 \times 10^{6} \mathrm{~Pa} . \mathrm{s}$, which is extremely high for the purpose of melt extrusion. The complex viscosity of the polymers decreased with the increase in temperature, reaching 12,000-14,000 Pa.s for Affinisol ${ }^{\mathrm{TM}}$ HPMC HME and 2000 Pa.s for Kollidon ${ }^{\circledR}$ VA 64 at $180^{\circ} \mathrm{C}$. It has been reported earlier that the viscosity of a polymer at an angular frequency of $0.1 \mathrm{rad} / \mathrm{s}$ should optimally be in the range of 10,000 to 1000 Pa.s to enable melt extrusion $(12,19)$. Such a range was established based on the following considerations: (i) the polymer should be fluid enough with low viscosity so that the drug could dissolve in it, (ii) the plasticity of the polymer should be maintained so the material flows through the barrel to be extruded at the other end and does not melt and settle on the bottom of the barrel leading to degradation, and (iii) the extrudates exit from the dies as strands that are amenable to down-stream processing. In other words, the viscosity of the polymer should be low enough for successful formation of solid dispersion and high enough for successful extrusion to take place. Therefore, based on the complex viscosity at $0.1 \mathrm{rad} / \mathrm{s}$, it appears that Affinisol ${ }^{\mathrm{TM}}$ HPMC HME polymers may require processing at $>180^{\circ} \mathrm{C}$. This is, however, contrary to the previous reports where it was claimed the polymers may be processed at much lower temperatures $(16,17)$. As will be described later, further studies were conducted to address this apparent contradiction.

Compared to Affinisol ${ }^{\mathrm{TM}}$ HPMC HME polymers, Kollidon ${ }^{\circledR}$ VA 64 had much higher complex viscosity at lower temperature $\left(<140^{\circ} \mathrm{C}\right)$. However, there was a much faster drop in its viscosity as a function of an increase in temperature, since Kollidon ${ }^{\circledR}$ VA 64 exhibited lower viscosity than that of Affinisol ${ }^{\mathrm{TM}} \mathrm{HPMC} \mathrm{HME}$ polymers at $>150^{\circ} \mathrm{C}$ (Fig. 5). The

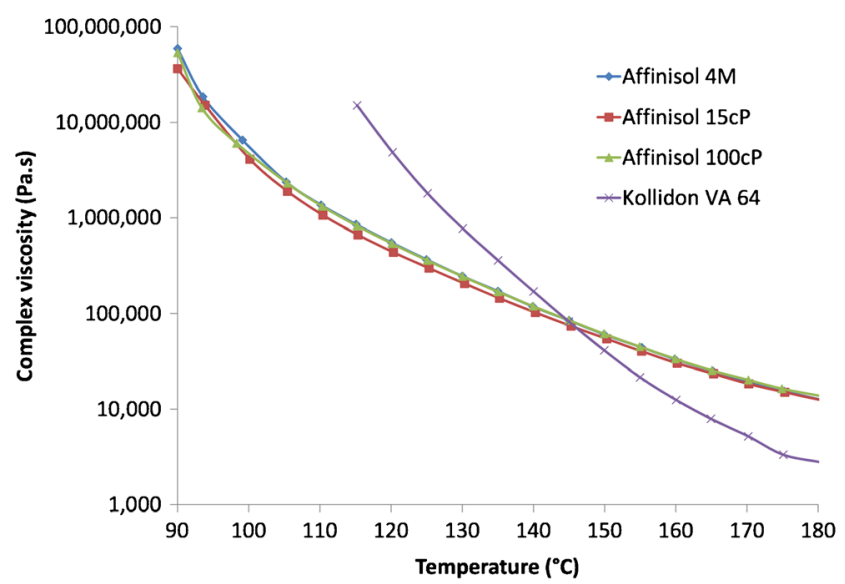

Fig. 5. Viscosities of Affinisol ${ }^{\mathrm{TM}}$ HPMC HME polymers and Kollidon ${ }^{\circledR}$ VA 64 as a function of temperature. Each data point represents the average of three determinations results with Kollidon ${ }^{\circledR}$ VA 64 are in agreement with a previous study, where it was shown that the polymer is extrudable above $157^{\circ} \mathrm{C}(13)$.

It should be noted here that the viscosity results reported in Fig. 5 are those of the polymers themselves in their softened or molten states, and they are not for $2 \%$ aqueous solutions, which are the values associated with their names as $15 \mathrm{cP}$, $100 \mathrm{cP}, 4 \mathrm{M}$ ( or $4000 \mathrm{cP}$ ), and so forth. While the viscosities of the aqueous solutions differed greatly, the melt viscosities were essentially similar. The latter are more relevant to melt extrusion.

\section{Effect of Angular Frequency on Complex Viscosity}

As mentioned earlier, the shear rate also has a major impact on the viscosity of polymers, especially if they exhibit shear-thinning nature. Such information is useful for hot melt extrusion since materials undergo high shear during processing due to rotating twin screws. Therefore, in order to conduct melt extrusion, not only the processing temperature of the barrel should be optimized, but also the effect of shear rate on the melt viscosity and, therefore, extrudability of the polymers should also be investigated. For this reason, the effect of shear rate on the polymers was studied in the present investigation by measuring their viscosity as a function of angular frequency. As shown in Fig. 6, a decrease in viscosity with an increase in angular frequency was observed for all polymers, indicating shear thinning. However, the extent of a decrease in viscosity with an increase in angular frequency at any particular temperature was different for different polymers. Among the three Affinisol ${ }^{\mathrm{TM}}$ polymers, Affinisol ${ }^{\mathrm{TM}} 15 \mathrm{cP}$ exhibited the highest complex viscosity (Fig. 6a), followed by Affinisol ${ }^{\mathrm{TM}}$ $100 \mathrm{cP}$ (Fig. 6b) and AffinisolTM 4 M (Fig. 6c). For Kollidon ${ }^{\circledR}$ VA 64, the viscosity was lower than that of Affinisol ${ }^{\mathrm{TM}}$ polymers at relatively lower angular frequencies, but the viscosity was relatively higher at higher frequencies (Fig. 6d), indicating that Kollidon ${ }^{\circledR}$ VA 64 has less shear thinning effect than that of the other polymers. In the log-log scale, the decrease in viscosity as a function of angular frequency at different temperatures was almost parallel. The decrease in viscosity for the polymers was also compared quantitatively by plotting viscosity as a function of angular frequency at a given temperature (Fig. 6e) and determining the slopes of the graphs. The following were the slopes for the polymers at $150^{\circ} \mathrm{C}$ : Affinisol ${ }^{\mathrm{TM}}$ $15 \mathrm{cP}=-0.563$, Affinisol ${ }^{\mathrm{TM}} 100 \mathrm{cP}=-0.547$, Affinisol ${ }^{\mathrm{TM}} 4 \mathrm{M}=$ -0.528 , and Kollidon ${ }^{\circledR}$ VA $64=-0.202$. It is clear that the drop in viscosity as a function of shear rate for Affinisol ${ }^{\mathrm{TM}} \mathrm{HME}$ polymers was relatively much faster than that of Kollidon ${ }^{\circledR}$ VA 64. Thus, during melt extrusion, the screw speed and screw design would have a more pronounced effect on the viscosity of AffinisolTM HPMC HME polymers and ultimately on the torque generated by them than that of Kollidon ${ }^{\circledR}$ VA 64 . Thus, it may be expected that Affinisol ${ }^{\mathrm{TM}}$ HPMC HME polymers could be processed at lower temperatures than the PVP-based polymer, while at the same time maintaining integrity of the extrudates during exit from the die.

It should be noted that the high shear rate exerted by the twin-screw extruders cannot be directly correlated with the viscosity measured by the rheometer as an angular frequency comparable to that of the shear rate of an extruder cannot be obtained in a rheometer. Despite the limitation of the 

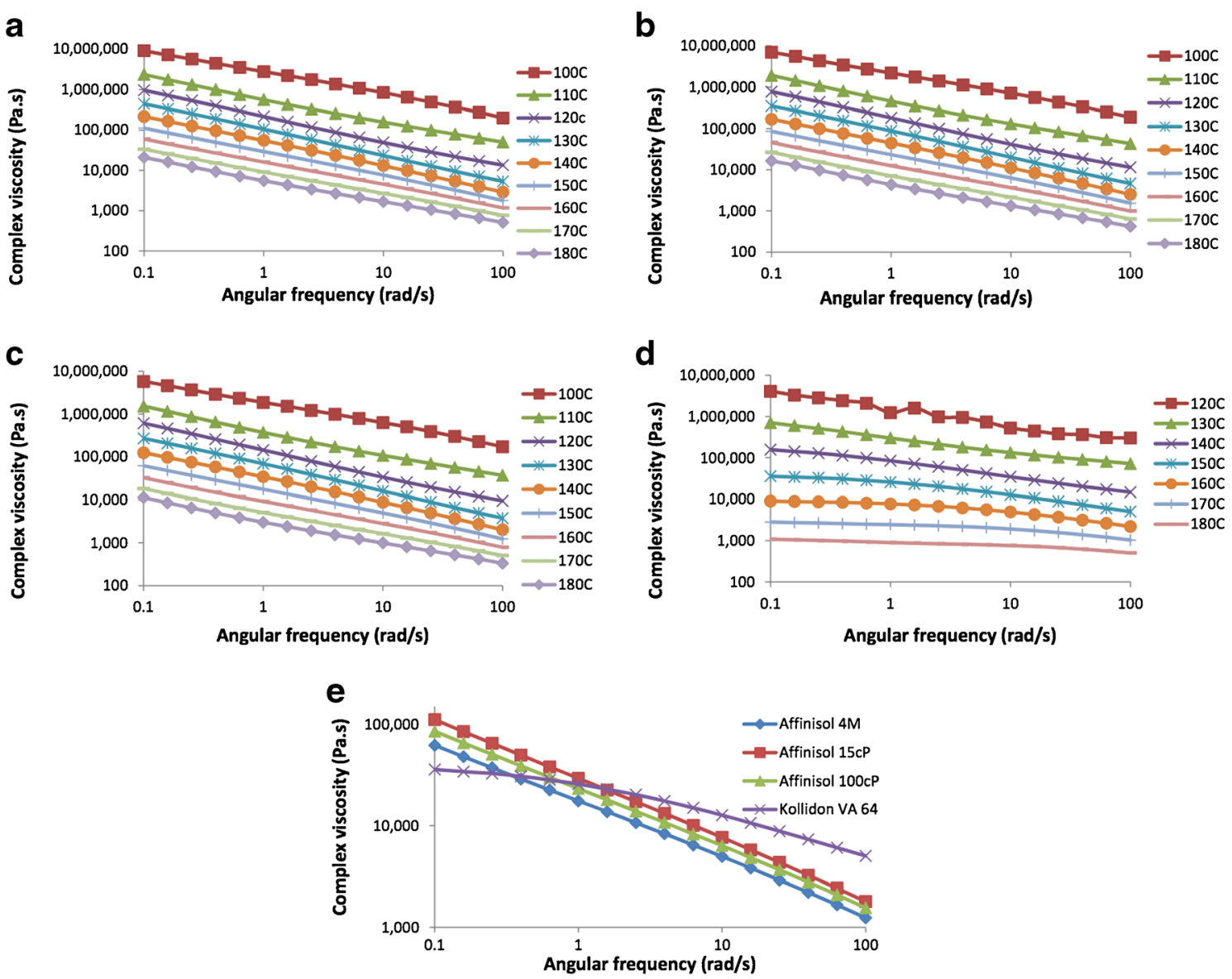

Fig. 6. Effect of angular frequency ( 0.1 to $100 \mathrm{rad} / \mathrm{s})$ and temperature on the complex viscosity: a Affinisol ${ }^{\mathrm{TM}} 15 \mathrm{cP}$, b Affinisol $^{\mathrm{TM}} 100 \mathrm{cP}, \mathbf{c}$ Affinisol ${ }^{\mathrm{TM}} 4 \mathrm{M}$, d Kollidon ${ }^{\circledR}$ VA 64 , and e comparison of the effect of angular frequency on the complex viscosity for different polymers at $150^{\circ} \mathrm{C}$

rheometer, the measured viscosity as a function of temperature gives a clear indication of how different polymers may behave during melt extrusion.

\section{Torque Analysis}

\section{Effect of Temperature and Screw Configuration}

The results of torque analysis of the three Affinisol ${ }^{\mathrm{TM}}$ HPMC HME polymers and Kollidon ${ }^{\circledR}$ VA 64 with three different screw designs and as a function of temperature are shown in Fig. 7. The force exerted on the twin screws by the material and processing parameters is referred to as torque. As mentioned earlier, the torque value of $12 \mathrm{Nm}$ in the Process 11 melt extruder is equivalent to $100 \%$ of the extruder capacity, above which the extruder stops. Therefore, the processing temperatures in the present study were selected such that the torque remained below $100 \%$ capacity. It was evident from our previous studies $(12,19)$ that the torque increased with a decrease in barrel temperature because of the increase in viscosity of the polymer. A similar trend was observed for the polymers used in the present study, except for Affinisol ${ }^{\mathrm{TM}}$ $4 \mathrm{M}$.

It is evident that during torque analysis the three Affinisol $^{\mathrm{TM}}$ polymers (Fig. 7a, b, and c) behave much differently than that of Kollidon ${ }^{\circledR}$ VA 64 (Fig. 7d). Kollidon ${ }^{\circledR}$ VA 64 exhibited acceptable torque values in a narrow temperature window for melt extrusion, and there was no major impact of screw configuration on torque generated during extrusion. For example, the torque generated by Kollidon ${ }^{\circledR}$ VA 64 was $54 \%$ at all the configurations at $180^{\circ} \mathrm{C}$, and it sharply increased to about $80 \%$ at a lower temperature of $160^{\circ} \mathrm{C}$. Below $160^{\circ} \mathrm{C}$, the material was practically nonextrudable by the equipment used.

Unlike Kollidon ${ }^{\circledR}$ VA 64, Affinisol ${ }^{\mathrm{TM}} 15 \mathrm{cP}$ (Fig. 7a) exhibited major effects of temperature and screw configuration on the torque generated during melt extrusion. Its torque was about $40 \%$ at $180^{\circ} \mathrm{C}$ at low stress screw design (L), which increased to $56 \%$ at medium stress $(\mathrm{M})$ and $63 \%$ at high stress $(\mathrm{H})$. There was a gradual increase in torque values as the stress levels on the materials are increased by changing the screw design. At $140^{\circ} \mathrm{C}$, the torque values for low, medium, and high stress screw designs were 75,80 , and $86 \%$, respectively. Thus, the polymer could not only be extruded at temperature wide range of temperature from 180 to $140^{\circ} \mathrm{C}$, its processing condition may also be varied by the screw design.

In case of Affinisol ${ }^{\mathrm{TM}} 100 \mathrm{cP}$ (Fig. 7b), the torque values at all three screw designs were essentially similar. The torque values at $180^{\circ} \mathrm{C}$ for low, medium, and high intensities were 54, 56 , and $60 \%$, respectively. They were still within the working 

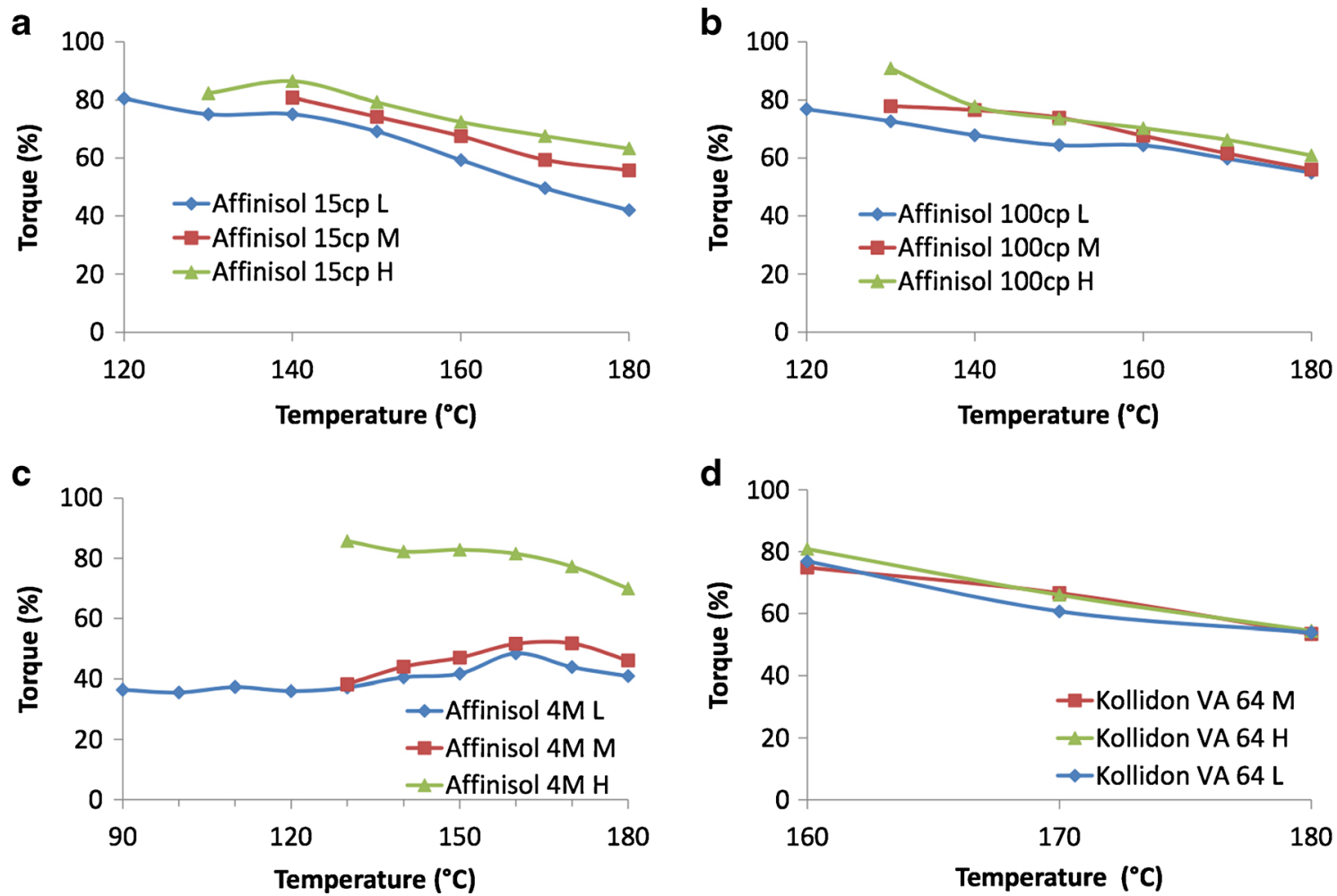

Fig. 7. Effect of melt extrusion processing temperature and screw configurations (low, L; medium, M; high, L) on torque observed during melt extrusion of different polymers: a Affinisol ${ }^{\mathrm{TM}} 15 \mathrm{cP}$, b Affinisol ${ }^{\mathrm{TM}} 100 \mathrm{cP}$, c Affinisol ${ }^{\mathrm{TM}} 4 \mathrm{M}$, and d Kollidon ${ }^{\circledR}$ VA 64

range of the melt extruder when the temperature was lowered to $140^{\circ} \mathrm{C}$, where torque values were $67 \%$ for low, $77 \%$ for medium, and $78 \%$ for high intensity screw designs.

The torque analysis data were significantly different in case of Affinisol ${ }^{\mathrm{TM}} 4 \mathrm{M}$ (Fig. 7c) than those of 15 and $100 \mathrm{cP}$ grades. At $180^{\circ} \mathrm{C}$, the torque values for Affinisol ${ }^{\mathrm{TM}} 4 \mathrm{M}$ at low, medium, and high intensities were 41,46 , and $70 \%$, respectively. With a decrease in temperature, there was a slight increase in torque to $86 \%$ at $130^{\circ} \mathrm{C}$ at the high stress screw design. On the other hand, there was small decrease in torque at low and medium intensities followed by a plateau. In case of both low and medium stress screw designs, the torque value was about $38 \%$ at $130^{\circ} \mathrm{C}$. At low stress screw design, the polymer could be extruded at a temperature as low as $120^{\circ} \mathrm{C}$ with a torque value of $36 \%$, below which the output material was granular in nature.

It should be noted here that different levels of shear stress, which is the force applied to the materials by twin screws, may be necessary in a melt extruder to enable adequate mixing or kneading of materials. High stress may also be needed for dissolution of drug in the polymer melt. In the present investigation, the shear stress was changed by using different screw designs. In the low stress screw design (L), kneading screws with $30^{\circ}$ angles were used, while the screws were partially changed to those with $60^{\circ}$ and $90^{\circ}$ angles in, respectively, medium $(\mathrm{M})$ and high $(\mathrm{H})$ shear screw designs. As the angles of screws were increased, they might have provided more resistance to the flow of materials within a melt extruder. This could be the reason for an increase in torque observed in Fig. 7 with the increase shear stress by screw design for Affinisol ${ }^{\mathrm{TM}} 15 \mathrm{cP}$ and Affinisol ${ }^{\mathrm{TM}} 100 \mathrm{cP}$ (Fig. 7a and b, respectively). Although the torque of Affinisol $^{\mathrm{TM}} 4 \mathrm{M}$ at high $(\mathrm{H})$ stress screw design was similar to those with Affinisol ${ }^{\mathrm{TM}} 15 \mathrm{cP}$ and Affinisol ${ }^{\mathrm{TM}} 100 \mathrm{cP}$, much lower torque was observed at $\mathrm{L}$ and $\mathrm{M}$ screw designs. The reason for this anomalous behavior was not investigated. The effect of screw design on the torque of Kollidon ${ }^{\circledR}$ VA 64 was negligible as it appeared that any resistance applied by the screw design to the flow of the material within the extruder was minimal.

It may also be mentioned here that the shear stress applied by screw design is different from the shear rate exerted on the materials during melt extrusion. In rheological analysis, the rate at which the oscillation of the parallel plate takes place (or angular frequency) is called the shear rate. According to the results presented in Fig. 6, the complex viscosity of polymers decreased with an increase in shear rate. A similar relationship was observed in one of our previous studies when the torque value decreased with an increase in screw speed (22). The shear rate was, however, kept constant in the present investigation as the screw speed and feed rate remained unchanged in all melt extrusion experiments. Therefore, any effects of screw designs observed in Fig. 7 are due to change in shear stress and not in shear rate.

\section{Effect of Angular Frequency on Torque: Affinisol ${ }^{\mathrm{TM}}$ HPMC HME Polymers vs. Kollidon ${ }^{\circledR}$ VA 64}

In Fig. 5, the complex viscosity of the Affinisol ${ }^{\mathrm{TM}}$ HPMC $\mathrm{HME}$ polymers at $0.1 \mathrm{rad} / \mathrm{s}$ was higher than $10,000 \mathrm{~Pa} . \mathrm{s}$ at 
temperatures as high as $180^{\circ} \mathrm{C}$. On the other hand, the viscosity of Kollidon ${ }^{\circledR}$ VA 64 was below 10,000 Pa.s at $\sim 160^{\circ} \mathrm{C}$. Since the complex viscosity should be in the range of 10,000 to $1000 \mathrm{~Pa}$.s for optimal melt extrudability $(12,22)$, these results suggest that it would be difficult, if not impossible, to extrude the Affinisol ${ }^{\mathrm{TM}}$ HPMC HME polymers through a melt extruder even at $180^{\circ} \mathrm{C}$, while Kollidon ${ }^{\circledR}$ VA 64 may be extruded at a lower temperature of $160^{\circ} \mathrm{C}$. On the contrary, the results of torque analysis in the present study (Fig. 7a, b, and c) demonstrate that the Affinisol ${ }^{\mathrm{TM}}$ HPMC HME polymers may be processed at a temperature as low as $130^{\circ} \mathrm{C}$ with all three screw designs, and in some cases, the temperature may be lowered even further. However, the lower limit of the temperature window for the processing of Kollidon ${ }^{\circledR}$ VA 64 still remained $\sim 160^{\circ} \mathrm{C}$. This apparent contradiction between viscosity and extrudability of Affinisol ${ }^{\mathrm{TM}}$ HPMC HME polymers and Kollidon ${ }^{\circledR}$ VA 64 may be explained by the difference in the effect of angular frequency on complex viscosity of the polymers (Fig. 6). The slopes of the complex viscosity $v s$. angular frequency plots for one of the Affinisol ${ }^{\mathrm{TM}}$ HPMC HME polymers (AffinisolTM HPMC HME $4 \mathrm{M}$ ) and Kollidon ${ }^{\circledR}$ VA 64 at different temperatures from 120 to $180^{\circ} \mathrm{C}$ (Fig. 6) were calculated, and the results are given in Table III. A $t$ test was performed to evaluate whether there was any significant difference between the slopes of the two polymers. The critical $t$ value was found to be $-11.5(d f=6)$ and $p$ value for two tail was $<0.05$. It could thus be concluded that the effect of shear rate on the decrease in viscosity for Affinisol $^{\text {TM }}$ HPMC HME $4 \mathrm{M}$ was significantly greater than that on Kollidon ${ }^{\circledR}$ VA 64 . Because of much higher slopes of the viscosity $v s$. angular frequency plots, the torque of AffinisolTM $^{\text {TM }}$ HC HME $4 \mathrm{M}$ and other similar polymers decreased sharply to extrudable ranges (Fig. 7a, b, and c). This is the direct result of the high shear rate exerted by the extruder. Consequently, the temperature window for the processing of AffinisolTM HPMC HME also became relatively much larger (Fig. 7a, b, and c). In contrast, the torque of Kollidon ${ }^{\circledR}$ VA 64 remained high, and the temperature window for its processing was practically unchanged (Fig. 7d) because of the lower slopes of its viscosity $v s$. the angular frequency plots (Fig. 6d and Table III). A similar correlation between the effect of angular frequency and torque was reported earlier by Rosalina et al. (23) for starch gels. It might be possible that Affinisol $^{\mathrm{TM}}$ HPMC HME polymers have gel-like structures in the molten state that are highly thixotropic and, therefore, exhibit shear thinning. Thus, the results of the present investigation demonstrate that for certain polymers, it is not only

Table III. Comparison of the Slopes of Oscillation Frequency vs. Complex Viscosity Curves for Kollidon ${ }^{\circledR}$ VA 64 and Affinisol ${ }^{\mathrm{TM}} 4 \mathrm{M}$

\begin{tabular}{lcc}
\hline Temperature $\left({ }^{\circ} \mathrm{C}\right)$ & Affinisol $^{\mathrm{TM}} 4 \mathrm{M}$ slope & Kollidon ${ }^{\circledR}$ VA 64 slope \\
\hline 120 & -0.612 & -0.404 \\
130 & -0.624 & -0.338 \\
140 & -0.596 & -0.358 \\
150 & -0.561 & -0.292 \\
160 & -0.531 & -0.202 \\
170 & -0.510 & -0.133 \\
180 & -0.502 & -0.099 \\
\hline
\end{tabular}

necessary to know the complex viscosity, but it is also important to study the effect of angular frequency on complex viscosity to fully characterize them for melt extrudability in the development of solid dispersion systems.

\section{Concluding Remarks}

DSC and PXRD analyses showed that the three AffinisolTM $^{\mathrm{TM}}$ HC HME polymers were amorphous in nature and may be used as suitable matrices to formulate amorphous drugs in solid dispersions. Their $\mathrm{T}_{\mathrm{g}}$ values were similar to that of Kollidon ${ }^{\circledR}$ VA 64 , which was previously studied for its suitability for hot melt extrusion $(12,22)$. TGA showed that the onset of degradation for all polymers was above $220^{\circ} \mathrm{C}$, which makes them viable candidates for HME processing at high temperatures. The Affinisol ${ }^{\mathrm{TM}}$ HPMC HME polymers exhibited less hygroscopicity than Kollidon ${ }^{\circledR}$ VA 64 and another comparable HPMC polymer, MethocelTM K100LV.

A decrease in complex viscosity was observed for all polymers with an increase in temperature. The viscosity $v s$. temperature plots were almost identical for Affinisol ${ }^{\mathrm{TM}}$ polymers, while the decrease in viscosity of Kollidon ${ }^{\circledR}$ VA 64 as a function of temperature was more rapid as it showed higher viscosity than that of Affinisol ${ }^{\mathrm{TM}}$ polymers at $<150^{\circ} \mathrm{C}$ and lower viscosity at $>150^{\circ} \mathrm{C}$. However, when a frequency sweep was conducted, it was observed that the decrease in viscosity for the Affinisol ${ }^{\mathrm{TM}}$ HPMC HME polymers was highly dependent on angular frequency or the shear rate applied to the polymers. A drop in viscosity as a function of angular frequency was plotted at $150^{\circ} \mathrm{C}$ for all four polymers. The influence of shear rate on the viscosity of Kollidon ${ }^{\circledR}$ VA 64 was the least, which was evident from the low slopes of the graphs. In case of Affinisol $^{\text {TM }}$ HPMC HME polymers, the viscosity of $15 \mathrm{cP}$ grade was the highest, followed by $100 \mathrm{cP}$ and then $4 \mathrm{M}$. Such effects of temperature and shear rate on the viscosity could be extrapolated to the hot melt extrusion process. Affinisol ${ }^{\mathrm{TM}}$ polymers showed larger windows of processing temperatures as compared to that of Kollidon ${ }^{\circledR}$ VA 64. This was due to a larger decrease in viscosity caused by high shear rate in case of Affinisol ${ }^{\mathrm{TM}}$ polymers as compared to Kollidon ${ }^{\circledR}$ VA 64 .

The properties of only the neat polymers were compared in the present investigation. However, for the selection of optimal polymers in the formulation of solid dispersion by melt extrusion, it is essential to understand the effects of drugs, fillers, or plasticizers on melt viscosity and extrudability of polymers. It is also important to investigate the relative miscibility of drugs in different polymers. In a recent investigation, a cellulosic polymer, HPMC phthalate, had about three times the miscibility as that of Kollidon ${ }^{\circledR}$ VA 64 (24). It will also be interesting to know how miscible Affinisol ${ }^{\mathrm{TM}}$ polymers are relative to Kollidon ${ }^{\circledR}$ VA 64 and other polymers. No such studies were conducted in the present investigation. We hope that the systematic characterization of Affinisol ${ }^{\mathrm{TM}}$ HPMC HME polymers in the presented in this report will lead to such studies in the future and facilitate their use in drug product development. 


\section{REFERENCES}

1. Pudipeddi M, Serajuddin ATM, Mufson D. Integrated drug product development-from lead candidate selection to life-cycle management. The Process of New Drug Discovery and Development. 2006:15.

2. Serajuddin ATM. Salt formation to improve drug solubility. Adv Drug Deliv Rev. 2007;59(7):603-16.

3. Dressman JB, Amidon GL, Reppas C, Shah VP. Dissolution testing as a prognostic tool for oral drug absorption: immediate release dosage forms. Pharm Res. 1998;15(1):11-22.

4. Li S, He H, Parthiban LJ, Yin H, Serajuddin ATM. IV-IVC considerations in the development of immediate-release oral dosage form. J Pharm Sci. 2005;94(7):1396-417.

5. Vasanthavada M, Tong W, Serajuddin A. Development of solid dispersion for poorly water-soluble drugs. Water-insoluble drug formulations. 2nd ed. New York: Informa Healthcare; 2008. p. 149-84.

6. Surikutchi BT, Patil SP, Shete G, Patel S, Bansal AK. Drugexcipient behavior in polymeric amorphous solid dispersions. J Excipients Food Chem. 2013;4(3):70-94.

7. Serajuddin ATM. Solid dispersion of poorly water-soluble drugs: early promises, subsequent problems, and recent breakthroughs. J Pharm Sci. 1999;88(10):1058-66.

8. Breitenbach J. Melt extrusion: from process to drug delivery technology. Eur J Pharm Biopharm. 2002;54(2):107-17.

9. Crowley MM, Zhang F, Repka MA, Thumma S, Upadhye SB, Kumar Battu S, et al. Pharmaceutical applications of hot-melt extrusion: part I. Drug Dev Ind Pharm. 2007;33(9):909-26.

10. Lakshman JP, Cao Y, Kowalski J, Serajuddin ATM. Application of melt extrusion in the development of a physically and chemically stable high-energy amorphous solid dispersion of a poorly water-soluble drug. Mol Pharm. 2008;5(6):994-1002.

11. Shah S, Maddineni S, Lu J, Repka MA. Melt extrusion with poorly soluble drugs. Int J Pharm. 2013;453(1):233-52.

12. Gupta SS, Meena A, Parikh T, Serajuddin AT. Investigation of thermal and viscoelastic properties of polymers relevant to hot melt extrusion, I: Polyvinylpyrrolidone and related polymers. J Excipients Food Chem. 2014;5(1):32-45.
13. Meena A, Parikh T, Gupta SS, Serajuddin AT. Investigation of thermal and viscoelastic properties of polymers relevant to hot melt extrusion, II: Cellulosic polymers. J Excipients Food Chem. 2014;5(1):46-55.

14. Parikh T, Gupta SS, Meena A, Serajuddin AT. Investigation of thermal and viscoelastic properties of polymers relevant to hot melt extrusion, III: polymethacrylates and polymethacrylic acid based polymers. J Excipients Food Chem. 2014;5(1):56-64.

15. Siew A. New excipient options for oral solid dosage drugs. PharmTech (online edition). January 30, 2015 (http:// www.pharmtech.com/new-excipient-options-oral-solid-dosage-drugs)

16. O'Donnell KP, Grasman N, Shrestha U, Vanchura B. The utility of AFFINISOL TM HPMC in Hot melt extrusion for supersaturation and controlled release of poorly soluble drugs. San Diego, CA: AAPS Annual Meeting; 2014.

17. Porter III W, O'Donnell K, Spaulding W, Shrestha U. Transition from spray drying to melt extrusion. Poster presented at the AIChE Annual meeting, Atlanta, GA, November 2014 (http:// www3.aiche.org/proceedings/Abstract.aspx?PaperID=386964)

18. Kolter H, Karl M, Grycke A. Hot-melt extrusion with BASF polymers: extrusion compendium. 2nd ed. Germany: BASF Corp; 2012. p. 113-6.

19. Nishiyami Y, Sugiyama J, Chanzy H, Langan P. Crystal structure and hydrogen bonding system in cellulose I $\alpha$ from synchrotron $\mathrm{X}$-ray and neutron fiber diffraction. J Am Chem Soc. 2003;125(47):14300-6.

20. Ahlneck C, Zografi G. The molecular basis of moisture effects on the physical and chemical stability of drugs in the solid state. Int J Pharm. 1990;62(2):87-95.

21. Akdogan H. Pressure, torque, and energy responses of a twin screw extruder at high moisture contents. Food Res Int. 1996;29(5-6):423-9.

22. Gupta SS, Parikh T, Meena AK, Mahajan N, Vitez I, Serajuddin ATM. Effect of carbamazepine on viscoelastic properties and hot melt extrudability of Soluplus ${ }^{\circledR}$. Int J Pharm. 2015;478(1):232-9.

23. Rosalina I, Bhattacharya M. Dynamic rheological measurements and analysis of starch gels. Carbohydr Polym. 2002;48(2):191-202.

24. Parikh T, Gupta SS, Meena AK, Vitez I, Mahajan N, Serajuddin ATM. Application of film-casting technique to Investigate drugpolymer miscibility in solid dispersion and hot-melt extrudate. J Pharm Sci. 2015;104(7):2142-52. 